\title{
Impact of atrial fibrillation in critically-ill patients admitted to a stepdown unit
}

Lorenzo Falsetti MD $\mathrm{PhD}^{1 *}$, Marco Proietti $\mathrm{MD} \mathrm{PhD}^{2,3 *}$, Vincenzo Zaccone $\mathrm{MD}^{1}$, Federico Guerra $\mathrm{MD}^{4}$, Cinzia Nitti MD ${ }^{1}$, Aldo Salvi MD${ }^{1}$, Giovanna Viticchi MD ${ }^{5}$, Francesca Riccomi $\mathrm{MD}^{6}$, Mattia Sampaolesi $\mathrm{MD}^{6}$, Mauro Silvestrini $\mathrm{MD}^{5}$, Gianluca Moroncini ${ }^{7} \mathrm{MD} \mathrm{PhD}$, Gregory Y.H. Lip ${ }^{8}$ MD, Alessandro Capucci ${ }^{4}$ MD

${ }^{1}$ Internal and Sub-intensive Medicine Department, A.O.U. “Ospedali Riuniti”, Ancona, Italy; ${ }^{2}$ Department of Clinical Sciences and Community Health, University of Milan, Milan, Italy; ${ }^{3}$ Liverpool Centre for Cardiovascular Science, University of Liverpool, Liverpool, United Kingdom; ${ }^{4}$ Cardiology and Arrhythmology Clinic, Department of Biomedical Sciences and Public Health, Marche Polytechnic University, A.O.U. "Ospedali Riuniti”, Ancona, Italy; ${ }^{5}$ Neurologic Clinic, Clinical and Experimental Medicine Department, Marche Polytechnic University, A.O.U. "Ospedali Riuniti”, Ancona, Italy; ${ }^{6}$ Emergency Medicine Residency Program, Marche Polytechnic University, Ancona, Italy; ${ }^{7}$ Clinica Medica, Azienda Ospedaliero-Universitaria "Ospedali Riuniti" di Ancona, Italy, Ancona, Italy; ${ }^{8}$ Liverpool Centre for Cardiovascular Science, University of Liverpool and Liverpool Heart \& Chest Hospital, Liverpool, United Kingdom

*both authors equally contributed to the paper.

Word Count: 2586

Corresponding Author:

Dr. Lorenzo Falsetti

ORCID: https://orcid.org/0000-0003-3411-2388 
Internal and Sub-intensive Medicine Department, A.O.U. “Ospedali Riuniti”, Ancona, Italy Via Conca 10, Ancona (Italy)

Tel: +38 071596 5269; Mobile: +39 338 7034188; email: drfalsetti@yahoo.it 


\section{ABSTRACT}

Background: limited data are available on the clinical course of patients with history of atrial fibrillation (AF) when admitted in an intensive care environment. We aimed to describe the occurrence of major adverse events in AF patients admitted to a stepdown care unit (SDU) and to analyse clinical factors associated with outcomes, impact of dicumarolic oral anticoagulant (OAC) therapy impact and performance of clinical risk scores in this setting.

Materials and Methods: Single-center, observational retrospective analysis on a population of subjects with AF history admitted to a SDU. Therapeutic failure (composite of transfer to ICU or death) was considered the main study outcome. Occurrence of stroke and major bleeding $(\mathrm{MH})$ were considered as secondary outcomes. The performance of clinical risk scores was evaluated.

Results: 1430 consecutive patients were enrolled. 194 (13.6\%) reported the main outcome. Using multivariate logistic regression, age (odds ratio $[\mathrm{OR}]: 1.03,95 \%$ confidence interval [CI]:1.01-1.05), acute coronary syndrome (OR:3.10,95\%CI:1.88-5.12), cardiogenic shock (OR:10.06,95\%CI:5.37-18.84), septic shock (OR:5.19,95\%CI:3.29-18.84), acute respiratory failure (OR:2.49,95\%CI:1.67-3.64) and $\mathrm{OAC}$ use (OR:1.61,95\%CI:1.02-2.55) were independently associated with main outcome. OAC prescription was associated with stroke risk reduction and to both $\mathrm{MH}$ and main outcome risk increase. $\mathrm{CHA}_{2} \mathrm{DS}_{2}-\mathrm{VASc}$ (cindex:0.545, $\mathrm{p}=0.117$ for stroke) and HAS-BLED (c-index:0.503, $\mathrm{p}=0.900$ for $\mathrm{MH}$ ) did not significantly predict events occurrence.

Conclusions: In critically-ill AF patients admitted to a SDU, adverse outcomes are highly prevalent. OAC use is associated to an increased risk of therapeutic failure, clinical scores seem unhelpful in predicting stroke and $\mathrm{MH}$, suggesting a highly individualized approach in AF management in this setting. 
Keywords: atrial fibrillation; critical care; outcomes; anticoagulation; clinical risk scores.

\section{INTRODUCTION}

Atrial fibrillation (AF) is the most common sustained arrhythmia observed in general population[1] and several groups of hospitalized patients[2]. Among critically-ill subjects admitted in intensive care units (ICU), pre-existing and new-onset AF can be observed in 1 out of 3 patients[3]. New-onset AF has a prevalence between $5-46 \%$ and represents $52 \%$ of the atrial arrhythmias in ICU[2,3]. Pre-existing AF follows the prevalence of the general population, and is present in $9 \%$ in critically-ill patients admitted to ICU[3].

AF is associated to an increased risk of stroke, cardiovascular events and cardiovascular or allcause death[1,4]. In the context of critically-ill patients, AF is associated to a deterioration of haemodynamic state[3], as well as an increased stroke risk[5], acute decompensated heart failure (AHF)[6] and death[7]. In hospitalized patients new-onset AF does not independently predict in-hospital death[8], pre-existing AF is an independent risk factor for in-hospital mortality and worse functional outcomes[9,10]. In order to evaluate the baseline thromboembolic and bleeding risk and to prevent stroke and major bleeding occurrence, clinical prognostic scores are commonly used[11]. Nevertheless, their role for risk stratification in critically-ill patients is debated[12].

Medical subjects at risk of clinical deterioration are admitted from emergency department to stepdown units (SDU) in order to optimize patient's care[13]. Main objective of this study was to evaluate therapeutic failure, defined as the composite of death or ICU transfer in criticallyill patients with pre-existing AF admitted to a SDU. Second, we evaluated thromboembolic and haemorragic events occurrence and their relationship with clinical prognostic scores. Finally, we evaluated the adherence to European Society of Cardiology (ESC) AF guidelines' 
recommendations[1] regarding oral anticoagulation with vitamin $\mathrm{K}$ antagonists (OAC) and its association with study outcomes.

\section{METHODS}

\section{Study Cohort and Baseline Characteristics}

To evaluate study objectives, we retrospectively analysed a cohort of critically-ill patients with pre-existing AF admitted to the internal medicine department of the Azienda OspedalieroUniversitaria "Ospedali Riuniti", Ancona, Italy. Since January 01 2002 the department adopted an electronic medical record system (eMRS) for inpatients' management that allowed us to interrogate the main database to select patients characterized by a specific diagnosis. All diagnoses in the eMRS are coded according to ICD-9-CM: we selected all consecutive patients admitted with a concurrent diagnosis of AF (ICD-9:427.31) from inception to August $03^{\text {rd }}$ 2007 , in order to optimize data collection and to obtain a homogenous population in terms of clinical management and antithrombotic drugs use. We obtained from the eMRS and from the detailed examination of discharge reports all the data regarding demographics, history of risk factors and comorbidities, admission diagnoses, concurrent clinical events and antithrombotic drugs use. The study was approved by the institutional review board (Prot.168/2018,June $\left.21^{\text {st }}, 2018\right)$.

The data that support the findings of this study are openly available in Mendeley Data at http://doi.org/10.17632/c87p293wpb.4[14].

\section{Atrial Fibrillation}

The eMR contained patient's history, his clinical course, electrocardiographic, echocardiographic and cardiac monitor data. We retrieved AF presence and type (paroxysmal, persistent or permanent) according to the definition current at the moment of the study. Patients developing AF during the hospitalization with a history of known paroxysmal AF were 
considered, whereas subjects developing new-onset AF were excluded. We excluded all stable patients admitted for a programmed cardioversion procedure for AF rhythm control.

\section{Study Population}

Our SDU, according to the common definition[13], admits patients from the emergency department if affected by severe medical pathologies requiring an intermediate level of care, such as continuous electrocardiographic monitoring, inotropic or vasopressor support, noninvasive ventilation and renal replacement therapy, but still not necessitating of invasive ventilation or ICU care.

The acute pathologies considered for SDU admission were AHF, acute coronary syndromes (ACS), acute respiratory failure (ARF), acute kidney injury (AKI), infections, acute neurologic disorders, septic shock (SS), cardiogenic shock (CS), alone or in combination. Syncope and trauma were considered only as complicating conditions of the above-mentioned medical pathologies.

\section{Study Outcomes and Concurrent Clinical Events}

The main study outcome was the composite of death occurrence during SDU stay or ICU transfer due to the worsening of clinical conditions, requiring a more intensive and invasive management according to the clinical evaluation of the attending physicians. We also evaluated these events separately. The occurrence of concurrent clinical events during the SDU stay was also reported, with a specific interest in reporting thromboembolic (incident stroke or transient ischemic attack [TIA]) and major bleeding events. Concurrent clinical events were clinically defined by the attending physicians.

\section{Prognostic Clinical Scores}

In order to analyse the predictive ability of prognostic clinical risk scores, $\mathrm{CHA}_{2} \mathrm{DS}_{2}-\mathrm{VASc}$ and HAS-BLED scores were calculated according to the original schemes[11]. High 
thromboembolic risk was defined as $\mathrm{CHA}_{2} \mathrm{DS}_{2}-\mathrm{VASc} \geq 2$, high bleeding risk as HAS-BLED $\geq 3[11]$.

\section{Guidelines Adherence}

Adherence to recommendations in terms of antithrombotic treatment was evaluated according to ESC guidelines[1]: patients' treatment with antiplatelet drugs and $\mathrm{OAC}$ was defined as adherent to recommendations, undertreatment or overtreatment.

\section{Statistical Analysis}

Continuous variables were reported as mean and standard deviation or median and interquartile range, according to a normal or non-normal distribution of values. The statistical difference between subgroups was evaluated with t-test and ANOVA or with Mann-Whitney U-test and Kruskal-Wallis. Categorical variables were reported as number percentages, differences were evaluated with $\chi^{2}$ test.

The association of the clinical factors with the main outcome was evaluated with logistic regression analysis. Demographic and clinical variable differences between patients with and without the main outcome were first assessed with univariate analysis; then all the variables associated with the main outcome with a $\mathrm{p} \leq 0.10$ were included in multivariate analysis.

The association between risk scores and adverse clinical events was performed with a logistic regression model adjusted for AF type and OAC use. Risk scores predictivity was expressed using c-index. Sensitivity, specificity, positive predictive value (PPV) and negative predictive value (NPV) were calculated according to high thromboembolic and high bleeding risks cutoffs.

The association between guidelines adherence and outcomes was analysed with a logistic regression model, adjusted for all clinical variables different between patients adherent and not adherent to guidelines and that were significantly associated at univariate analysis. We 
considered as statistically significant a $p$-value $\leq 0.05$ for two-tailed tests. Statistical analyses were performed with SPSS 25.0 (IBM, USA).

\section{RESULTS}

1705 consecutive patients were retrieved through eMRS. After excluding those admitted for a planned cardioversion procedure, we included 1430 patients. From this cohort, we recorded 194(13.6\%) composite outcome events, including 160 deaths (11.2\%) and 34 ICU transfers $(2.4 \%)$

Baseline characteristics according to the occurrence of the composite outcome are summarised in Table 1. Patients reporting the composite outcome were significantly older, less burdened by hypertension and mitral valve disease (Table 1, Panel A and B), had less syncope and trauma but more ACS, CS, SS, ARF and infections than those without (Table 1, Panel C). Both admission systolic and diastolic blood pressures were significantly lower in those reporting the composite outcome (both $\mathrm{p}<0.001$ ) and, in a subgroup where data about left ventricular function were available, reduced function was associated with composite outcome $(p<0.001)$ (Table 1).

Patients experiencing the composite outcome were more treated with anticoagulants, more likely OAC than low-molecular weight heparin (LMWH), and less treated with antiplatelet drugs than those that did not experience the outcome (Table 1, Panel D). Subjects sustaining the composite outcome were less likely treated with angiotensin converting enzyme inhibitors/angiotensin receptor blockers $(\mathrm{p}<0.001)$ and class IC antiarrhythmic $\operatorname{drugs}(\mathrm{p}=0.008)$ but were more likely treated with inotropic drugs $(\mathrm{p}<0.001)$ (Table 1, Panel E).

The final multivariate logistic regression model found that age (odds ratio [OR]:1.03,95\% confidence interval[CI]:1.01-1.05), ACS $\quad(\mathrm{OR}: 3.10,95 \% \mathrm{CI}: 1.88-5.12), \quad \mathrm{CS}$ (OR:10.06,95\%CI:5.37-18.84), SS (OR:5.19,95\% CI: 3.29-18.84), ARF (OR:2.49,95\% CI:1.67-3.64) and OAC (OR:1.61,95\% CI:1.02-2.55) were associated with the occurrence of 
composite outcome (Table 2). Conversely, hypertension, mitral valve disease, admission diastolic blood pressure and antiplatelet drugs use were inversely associated with the composite outcome (Table 2).

\section{Guidelines Recommendation Adherence and Relationship with Outcomes}

According to ESC 2016 guidelines, 642(44.9\%) patients were guideline-adherent, 540 (37.8\%) were undertreated and $24(17.3 \%)$ were overtreated. Stroke/TIA was lowest in guidelineadherent patients $(\mathrm{p}<0.001)$ [Figure 1], but prevalence of both major bleeding and composite outcome was higher $(\mathrm{p}<0.001$ and $\mathrm{p}=0.020$, respectively)[Figure 1]. The final multivariable model (Table 3,Panel A) showed that undertreatment was associated with an increased stroke/TIA risk, with an inverse association with both major bleeding and composite outcome. Overtreatment only showed a nonsignificant trend for stroke/TIA (Table 3,Panel A).

\section{Thromboembolic and Bleeding Risks and Relationship with Outcomes}

At baseline, there was no difference in terms of thromboembolic risk according to $\mathrm{CHA}_{2} \mathrm{DS}_{2}$ VASc score between patients that experienced the composite outcome and those that did not experience it. HAS-BLED score, as well the proportion of patients with high bleeding risk were lower (both $\mathrm{p}<0.001$ ) in patients that reported the composite outcome (Table 1,Panel F). No significant difference was found in the distribution of the composite outcome according to $\mathrm{CHA}_{2} \mathrm{DS}_{2}$-VASc score $(\mathrm{p}=0.501)$. Conversely, the composite outcome occurred more frequently in patients with a lower HAS-BLED score $(\mathrm{p}<0.001)$.

Examination of stroke/TIA prevalence according to $\mathrm{CHA}_{2} \mathrm{DS}_{2}$-VASc score and major bleeding prevalence according to HAS-BLED score found no significant differences (Table 3, Panel B). The predictive ability of the two scores regarding the respective events was non-significant (CHA ${ }_{2} \mathrm{DS}_{2}$-VASc:0.545;95\%CI:0.489-0.601; HAS-BLED:0.503;95\%CI:0.453-0.554).

Considering high thromboembolic risk, we found high sensitivity (93.4\%,95\%CI:90.9-99.0\%), high NPV (95.0\%,95\%CI:87.6-98.1\%) and low specificity and PPV (Table 3, Panel B) of 
$\mathrm{CHA}_{2} \mathrm{DS}_{2}$-VASc $\geq 2$ for stroke/TIA. Regarding bleeding risk, intermediate values for both sensitivity $(46.6 \%, 95 \% \mathrm{CI}: 37.9-55.5 \%)$ and specificity $(57.1 \%, 95 \% \mathrm{CI}: 54.4-59.8 \%)$ were found, while high NPV (91.3\%,95\%CI:89.8-92.5\%) and very low PPV were reported (Table 3, Panel B).

\section{DISCUSSION}

In this study we found that in a cohort of critically-ill patients with a diagnosis of AF admitted to a SDU the composite outcome of death and ICU transfer was fairly prevalent, usually amongst older patients characterized by a worse and more severe clinical status. OAC use was associated to an increased risk of the composite outcome, while no impact of LMWH and an inverse association with antiplatelet drugs use was found. Guidelines adherence was associated with a stroke/TIA risk reduction, while undertreatment was inversely associated with the occurrence of major bleeding and the composite outcome. $\mathrm{CHA}_{2} \mathrm{DS}_{2}-\mathrm{VASc}$ and HAS-BLED scores were not associated with the stroke/TIA and major bleeding occurrence, nor showed significant predictive ability for these events; both scores demonstrated the ability of identifying patients with a very low risk.

The issue of new-onset AF in patients admitted to intensive care environment has been largely investigated and reported[15]. Several clinical risk factors, both related to the previous medical history and the severity of the acute condition, were positively associated to AF occurrence[15]. New-onset AF has been found associated with short- and long-term adverse outcomes[3].

Conversely, only scarce evidence exists in relation to the relative impact of pre-existing AF on the risk of adverse outcomes in ICU[8]. Indeed, our study provides the first European perspective about the high risk of major adverse events in critically-ill patients with preexisting AF. The evidence of $13.6 \%$ of patients reporting death or being transferred to the ICU 
requires careful consideration given the implications in terms of its impact on patients and healthcare resource use.

Our results are strengthened by a previous study, which reported a similar rate of major adverse events among a US cohort of patients admitted to medical ICU, similar to that of the SDU[8]. AF patients have an increased risk of death[16], but we underlined how in this context the risk of death is not associated to any of the classical AF-related risk factors rather than to the acute illness and depends on the overall clinical complexity of patients managed in this highly specific setting, as acute coronary syndrome, cardiogenic shock, acute respiratory failure, all conditions associated with a known increased thromboembolic and bleeding risks.

One of the most complex issues in the intensive care setting related to the management of OAC therapy. In a study performed in the Quebec region, considering patients with acute illnesses such as ACS, ARF or sepsis that developed AF, OAC use was not associated with a clinical benefit in terms of stroke risk reduction, but increased significantly the bleeding risk, especially in patients with a high bleeding risk (HAS-BLED $\geq 3$ )[17]. Several viewpoints emphasize the difficulty of taking the decision whether or not to prescribe OAC in ICU patients who developed AF, and it may be more relevant to consider OAC after ICU discharge, particularly for those with high thromboembolic risk[3]. Of note, ICU patients with new-onset AF also have an increased long-term risk for stroke and death[18]. Results of the multivariate regression analysis presented raise serious concerns about keeping anticoagulation in AF patients when admitted to intensive care. Conversely, LMWH use seems not to carry further risk, while antiplatelet drugs use seems associated to a lower risk of composite outcome. Even though we showed only an association, since the study design did not allow to provide any causal inference, we can postulate that the known anti-inflammatory effect of antiplatelet drugs[19] could be implied in the inverse relationship with the composite outcome we described. 
Several studies have addressed the relationship between guidelines adherence and improved outcomes in AF patients[20]. If, expectedly, those patients prescribed as undertreated showed an association with an increased stroke risk and lower major bleeding risk, the inverse association described with the composite outcome underlines how maintaining $\mathrm{OAC}$ in $\mathrm{AF}$ during SDU staying could not be indicated. In the context of intensive care, we are not able to provide specific "general" recommendations on how to manage thromboembolic risk in AF patients, rather a careful consideration of clinical status and an individual risk assessment. Specific studies in this context are clearly demanded to elucidate what would be the best approach.

$\mathrm{CHA}_{2} \mathrm{DS}_{2}$-VASc and HAS-BLED scores are validated and used to evaluate thromboembolic and bleeding risk in AF patients[21], being associated and able to predict the occurrence of death in AF patients[22]. Nevertheless, validation of these scores is lacking in ICU. Despite the high rate of reported events, our data show that $\mathrm{CHA}_{2} \mathrm{DS}_{2}-\mathrm{VASc}$ and HAS-BLED are unable to predict stroke and major bleeding. The high NPV documented for both the scores confirms their ability to identify 'low risk' patients. In a recent systematic review of the evidence available about use of scores in stroke and major bleeding prediction, all available risk scores report an overall moderate predictive ability, with the $\mathrm{CHA}_{2} \mathrm{DS}_{2}$-VASc and HASBLED the best option in the daily clinical practice[21]. Clinical scores have c-indexes approximately around 0.67 , hence their use cannot be absolutized and replace the physicians' clinical judgement and appropriate clinical assessment[21]. In this context, both scores were unable to predict adverse events in intensive care AF patients, and this underlines how clinical complexity overcomes the usual pathophysiological processes that lead to stroke and major bleeding, making it more unpredictable.

In order to correctly interpret our findings is important to make one important consideration. Both $\mathrm{CHA}_{2} \mathrm{DS}_{2}$-VASc and HAS-BLED scores were originally developed from general AF 
patients' cohorts and tested on 1-year outcomes[23,24]. Notwithstanding, since then both the scores were largely validated in various cohorts and tested on variable follow-up times[25-27]; indeed the use of both the scores are currently recommended from most of the international clinical AF guidelines[28].

\section{Limitations}

The main limitation is related to the study design, being a retrospective observational analysis of a cohort not primarily identified for research purposes. Second, in the cohort of patients analysed only vitamin $\mathrm{K}$ antagonists were used as OAC. Third, we evaluated adherence to the application of current indications for anticoagulant therapy in a retrospective cohort, when the current reference guidelines had not been published, even though this was done exclusively exploratorily, allowed us to project the current guidelines recommendations to our cohort. While these limitations need to be accounted extending our findings to the overall AF population, the evidence we provided brings relevant implications in terms of clinical management of AF patients admitted to SDU and ICU.

\section{CONCLUSIONS}

In critically-ill AF patients admitted to a SDU, adverse outcomes are highly prevalent. OAC use is associated to an increased risk for the composite outcome, even in those patients correctly prescribed according to guidelines recommendations. Clinical scores may be unhelpful in predicting stroke and major bleeding occurrence, suggesting a highly individualized approach in evaluating the risk of adverse events and in the decision-making process of prescribing OAC therapy.

\section{Acknowledgments: None}

Funding: None

Conflict of Interest: None declared 
Authors' Contributions: LF,MP had full access to all of the data in the study and take responsibility for the integrity of the data and the accuracy of the data analysis. VZ,FG,CN,AS,VG,MS,FR,MS,GYHL,AC contributed substantially to the study design, data analysis and interpretation, and the writing of the manuscript. 


\section{REFERENCES}

1. Kirchhof P, Benussi S, Kotecha D, Ahlsson A, Atar D, Casadei B, et al. 2016 ESC Guidelines for the management of atrial fibrillation developed in collaboration with EACTS. Eur Heart J [Internet]. 2016 Oct 7 [cited 2017 Feb 28];37(38):2893-962. Available from: https://academic.oup.com/eurheartj/articlelookup/doi/10.1093/eurheartj/ehw210

2. Reinelt P, Karth GD, Geppert A, Heinz G. Incidence and type of cardiac arrhythmias in critically ill patients: a single center experience in a medical-cardiological ICU. Intensive Care Med. 2001 Sep;27(9):1466-73.

3. Bosch NA, Cimini J, Walkey AJ. Atrial Fibrillation in the ICU. Chest. 2018 Apr;154(6):1424-34.

4. Boriani G, Proietti M, Laroche C, Diemberger I, Popescu MI, Riahi S, et al. Changes to oral anticoagulant therapy and risk of death over a 3-year follow-up of a contemporary cohort of European patients with atrial fibrillation final report of the EURObservational Research Programme on Atrial Fibrillation (EORP-AF) pilot general r. Int J Cardiol [Internet]. 2018 Nov;271:68-74. Available from: https://linkinghub.elsevier.com/retrieve/pii/S0167527317380592

5. Walkey AJ, Wiener RS, Ghobrial JM, Curtis LH, Benjamin EJ. Incident stroke and mortality associated with new-onset atrial fibrillation in patients hospitalized with severe sepsis. Jama. 2011/11/15. 2011;306(20):2248-54.

6. Kanji S, Williamson DR, Yaghchi BM, Albert M, McIntyre L, Canadian Critical Care Trials Group. Epidemiology and management of atrial fibrillation in medical and noncardiac surgical adult intensive care unit patients. J Crit Care. 2012 Jun;27(3):326.e1-326.e8.

7. Bosch NA, Cimini J, Walkey AJ. Atrial Fibrillation in the ICU. Chest. 2018/04/09. 
2018 Apr;154(6):1424-34.

8. Carrera P, Thongprayoon C, Cheungpasitporn W, Iyer VN, Moua T. Epidemiology and outcome of new-onset atrial fibrillation in the medical intensive care unit. J Crit Care. 2016/11/05. 2016;36:102-6.

9. DiMarco JP. Atrial fibrillation and acute decompensated heart failure. Circ Heart Fail. 2009 Jan;2(1):72-3.

10. Gamst J, Christiansen CF, Rasmussen BS, Rasmussen LH, Thomsen RW. Pre-existing atrial fibrillation and risk of arterial thromboembolism and death in intensive care unit patients: a population-based cohort study. Crit Care. 2015 Aug;19(1):299.

11. Lip GYH, Lane DA. Stroke Prevention in Atrial Fibrillation. JAMA [Internet]. 2015 May 19 [cited 2015 May 19];313(19):1950-62. Available from: http://www.ncbi.nlm.nih.gov/pubmed/25988464

12. Guerra F, Scappini L, Maolo A, Campo G, Pavasini R, Shkoza M, et al. CHA2DS2VASc risk factors as predictors of stroke after acute coronary syndrome: A systematic review and meta-analysis. Eur Hear J Acute Cardiovasc Care. 2016 Oct;

13. Prin M, Wunsch H. The role of stepdown beds in hospital care. Am J Respir Crit Care Med. 2014 Dec;190(11):1210-6.

14. AFICILL Database. 2019 Dec;4.

15. McIntyre WF, Um KJ, Cheung CC, Belley-Côté EP, Dingwall O, Devereaux PJ, et al. Atrial fibrillation detected initially during acute medical illness: A systematic review. Eur Hear J Acute Cardiovasc Care. 2018;8(2):130-41.

16. Proietti M, Laroche C, Opolski G, Maggioni AP, Boriani G, Lip GYH, et al. "Realworld" atrial fibrillation management in Europe: observations from the 2-year followup of the EURObservational Research Programme-Atrial Fibrillation General Registry Pilot Phase. Europace [Internet]. 2017 May 18 [cited 2016 Jun 29];19(5):722-33. 
Available from: http://www.ncbi.nlm.nih.gov/pubmed/27194538

17. Quon MJ, Behlouli H, Pilote L. Anticoagulant Use and Risk of Ischemic Stroke and Bleeding in Patients With Secondary Atrial Fibrillation Associated With Acute Coronary Syndromes, Acute Pulmonary Disease, or Sepsis. JACC Clin Electrophysiol. 2018 Sep;4(3):386-93.

18. Kim K, Yang P-S, Jang E, Yu HT, Kim T-H, Uhm J-S, et al. Long-term impact of newly diagnosed atrial fibrillation during critical care: A Korean nationwide cohort study. Chest [Internet]. 2019 Apr 30 [cited 2019 May 4]; Available from: https://www.sciencedirect.com/science/article/pii/S0012369219308761

19. Pitchford SC. Novel uses for anti-platelet agents as anti-inflammatory drugs. Br J Pharmacol [Internet]. 2009 Jan 29 [cited 2019 May 5];152(7):987-1002. Available from: http://www.ncbi.nlm.nih.gov/pubmed/17603547

20. Lip GYH, Laroche C, Popescu MI, Rasmussen LH, Vitali-Serdoz L, Dan GA, et al. Improved outcomes with European Society of Cardiology guideline-adherent antithrombotic treatment in high-risk patients with atrial fibrillation: A report from the EORP-AF General Pilot Registry. Europace [Internet]. 2015 Aug 30 [cited 2015 Sep 3];17(12):1777-86. Available from: http:/www.ncbi.nlm.nih.gov/pubmed/26321406

21. Borre ED, Goode A, Raitz G, Shah B, Lowenstern A, Chatterjee R, et al. Predicting Thromboembolic and Bleeding Event Risk in Patients with Non-Valvular Atrial Fibrillation: A Systematic Review. Thromb Haemost [Internet]. 2018 Dec 30 [cited 2018 Dec 7];118(12):2171-87. Available from: http://www.thiemeconnect.de/DOI/DOI?10.1055/s-0038-1675400

22. Proietti M, Farcomeni A, Romiti GF, Di Rocco A, Placentino F, Diemberger I, et al. Association between clinical risk scores and mortality in atrial fibrillation: Systematic review and network meta-regression of 669,000 patients. Eur J Prev Cardiol [Internet]. 
2018 Dec 6 [cited 2018 Dec 7];204748731881766. Available from:

http://journals.sagepub.com/doi/10.1177/2047487318817662

23. Lip GYH, Nieuwlaat R, Pisters R, Lane DA, Crijns HJGM. Refining clinical risk stratification for predicting stroke and thromboembolism in atrial fibrillation using a novel risk factor-based approach: the euro heart survey on atrial fibrillation. Chest [Internet]. 2010 Feb [cited 2014 Jul 10];137(2):263-72. Available from: http://www.ncbi.nlm.nih.gov/pubmed/19762550

24. Pisters R, Lane DA, Nieuwlaat R, de Vos CB, Crijns HJGM, Lip GYH. A novel userfriendly score (HAS-BLED) to assess 1-year risk of major bleeding in patients with atrial fibrillation: the Euro Heart Survey. Chest [Internet]. 2010 Nov [cited 2014 Oct 17];138(5):1093-100. Available from: http://www.ncbi.nlm.nih.gov/pubmed/20299623

25. van Doorn S, Debray TPA, Kaasenbrood F, Hoes AW, Rutten FH, Moons KGM, et al. Predictive performance of the CHA2DS2-VASc rule in atrial fibrillation: a systematic review and meta-analysis. J Thromb Haemost [Internet]. 2017 Jun [cited 2018 Oct 17];15(6):1065-77. Available from: http://www.ncbi.nlm.nih.gov/pubmed/28375552

26. Joundi RA, Cipriano LE, Sposato LA, Saposnik G. Ischemic Stroke Risk in Patients With Atrial Fibrillation and CHA2DS2-VASc Score of 1: Systematic Review and Meta-Analysis [Internet]. Vol. 47, Stroke. Stroke; 2016 [cited 2020 Apr 27]. p. 13647. Available from: https://pubmed.ncbi.nlm.nih.gov/27026630/

27. Chang G, Xie Q, Ma L, Hu K, Zhang Z, Mu G, et al. Accuracy of HAS-BLED and other bleeding risk assessment tools in predicting major bleeding events in atrial fibrillation: A network meta-analysis. J Thromb Haemost [Internet]. 2020 Apr 1 [cited 2020 Apr 27];18(4):791-801. Available from:

https://onlinelibrary.wiley.com/doi/abs/10.1111/jth.14692 
28. Proietti M, Lane DA, Boriani G, Lip GYH. Stroke Prevention, Evaluation of Bleeding Risk, and Anticoagulant Treatment Management in Atrial Fibrillation Contemporary International Guidelines. Can J Cardiol [Internet]. 2019 May [cited 2019 Mar 10];35(5):619-33. Available from:

https://linkinghub.elsevier.com/retrieve/pii/S0828282X19301163 


\section{FIGURE LEGENDS}

Figure 1: Rate of Major Adverse Outcomes according to Adherence to Guidelines

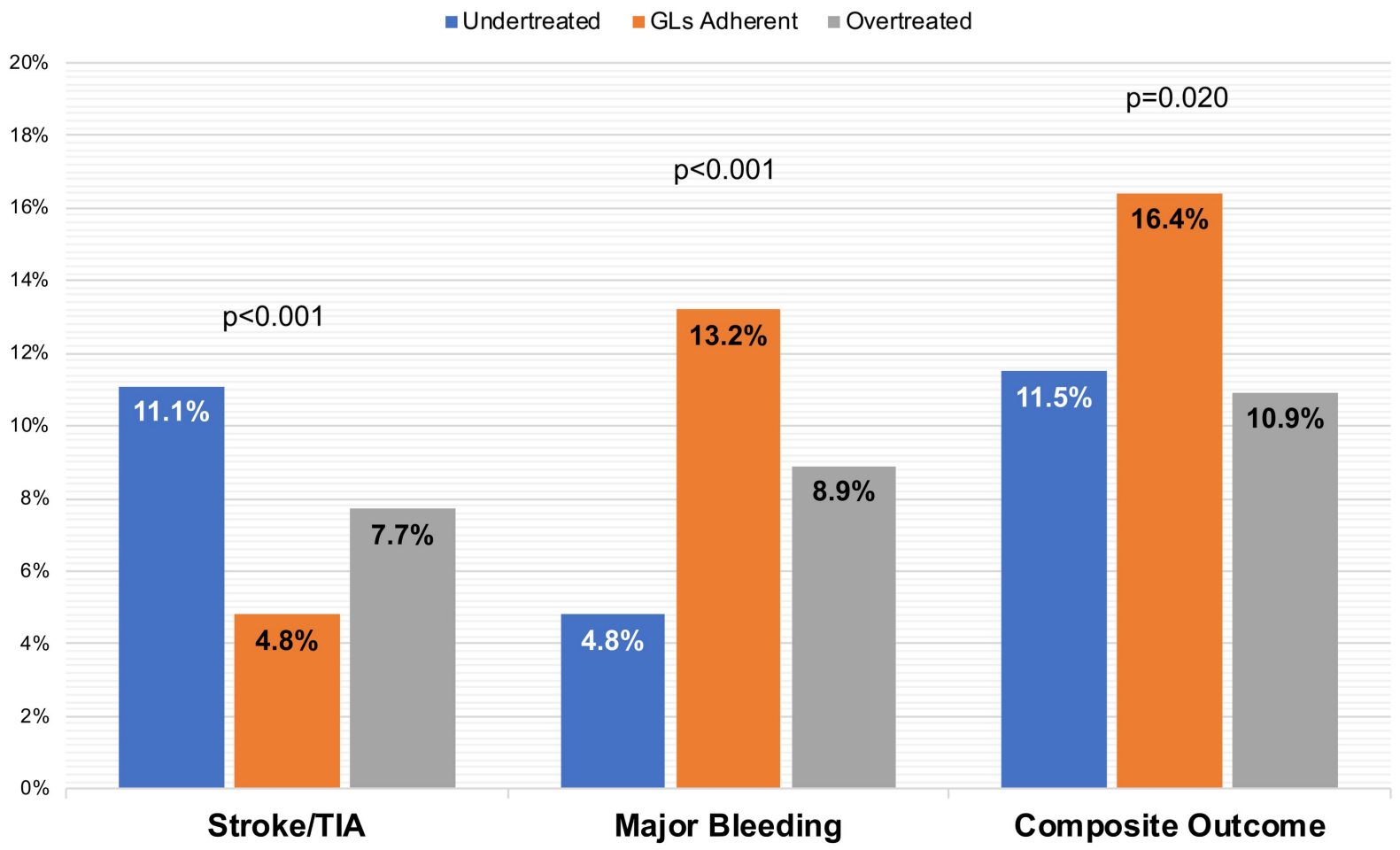

Legend: GLs $=$ Guidelines, TIA $=$ Transient ischemic attack 
Table 1: Baseline Characteristics at Admission in Sub-Intensive Unit (cont.)

\begin{tabular}{|c|c|c|c|}
\hline & \multicolumn{2}{|c|}{ Composite Outcome } & \multirow[t]{3}{*}{$\mathbf{p}$} \\
\hline & No & Yes & \\
\hline & $\mathrm{N}=1236$ & $\mathrm{~N}=194$ & \\
\hline \multicolumn{4}{|l|}{ A) Demographics } \\
\hline Age, years median [IQR] & $81[75-85]$ & 83 [77-89] & $<0.001$ \\
\hline Female Sex, n (\%) & $753(49.9)$ & $90(46.2)$ & 0.329 \\
\hline Type of AF, n (\%) & & & 0.077 \\
\hline Paroxysmal & $220(18.6)$ & $24(12.8)$ & \\
\hline Persistent & $292(24.7)$ & $57(30.3)$ & \\
\hline Permanent & $668(56.6)$ & $107(56.9)$ & \\
\hline SBP, $m m H g$ median [IQR] & $130[110-140]$ & 90 [80-122] & $<0.001$ \\
\hline DBP, $m m H g$ median [IQR] & $80[70-80]$ & $60[40-80]$ & $<0.001$ \\
\hline LV Function, n (\%) 524 & & & 0.001 \\
\hline Preserved & $326(67.4)$ & $17(42.5)$ & \\
\hline Reduced & $158(32.6)$ & $23(57.5)$ & \\
\hline
\end{tabular}

\section{B) Previous Clinical History}

$\begin{array}{lccc}\text { Hypertension, n (\%) } & 637(51.5) & 64(33.0) & <0.001 \\ \text { Diabetes Mellitus, n (\%) } & 226(18.3) & 34(17.5) & 0.799 \\ \text { Anaemia, n (\%) } & 114(9.2) & 17(8.8) & 0.836 \\ \text { Coronary Artery Disease, n (\%) } & 531(43.0) & 78(40.2) & 0.471 \\ \text { Peripheral Artery Disease, } \mathrm{n}(\%) & 131(10.6) & 16(8.2) & 0.316\end{array}$




\begin{tabular}{|c|c|c|c|}
\hline Chronic Heart Failure, n (\%) & $581(47.0)$ & $88(45.4)$ & 0.669 \\
\hline pCVP, n (\%) & $183(14.8)$ & $27(13.9)$ & 0.745 \\
\hline eCVP, n (\%) & $29(2.3)$ & $1(0.5)$ & 0.098 \\
\hline Stroke/TIA, n (\%) & $258(20.9)$ & $34(17.5)$ & 0.282 \\
\hline Mitral Disease, $\mathrm{n}(\%)$ & $195(15.8)$ & $15(7.7)$ & 0.003 \\
\hline Aortic Disease, n (\%) & $151(12.2)$ & $16(8.2)$ & 0.109 \\
\hline COPD, n (\%) & $340(27.5)$ & $54(27.8)$ & 0.925 \\
\hline Hepatic Disease, n (\%) & $35(2.8)$ & $7(3.6)$ & 0.551 \\
\hline CKD, n (\%) & $230(18.6)$ & $39(20.1)$ & 0.620 \\
\hline Previous Major Bleeding, $\mathrm{n}(\%)$ & $66(5.3)$ & $5(2.6)$ & 0.100 \\
\hline Neoplasm, n (\%) & $210(17.0)$ & $44(22.7)$ & 0.054 \\
\hline Comorbidities, $n$ median [IQR] & $3[2-4]$ & $2[2-3]$ & 0.114 \\
\hline \multicolumn{4}{|l|}{ C) Concurrent Clinical Events } \\
\hline Stroke/TIA, n (\%) & $90(7.3)$ & $20(10.3)$ & 0.141 \\
\hline Major Bleeding, n (\%) & $110(8.9)$ & $23(11.9)$ & 0.188 \\
\hline Syncope, n (\%) & $68(5.5)$ & $2(1.0)$ & 0.007 \\
\hline Acute Neurologic Disorders, n (\%) & $47(3.8)$ & $6(3.1)$ & 0.627 \\
\hline Trauma, n (\%) & $78(6.3)$ & $5(2.6)$ & 0.039 \\
\hline ACS, n (\%) & $160(12.9)$ & $50(25.8)$ & $<0.001$ \\
\hline Acute Heart Failure, n (\%) & $669(54.1)$ & $99(51.0)$ & 0.421 \\
\hline Cardiogenic Shock, n (\%) & $28(2.3)$ & $43(22.2)$ & $<0.001$ \\
\hline Septic Shock, n (\%) & $106(8.6)$ & $74(38.1)$ & $<0.001$ \\
\hline
\end{tabular}


Table 1: Baseline Characteristics at Admission in Sub-Intensive Unit (cont.)

\begin{tabular}{lccr}
\hline AKI, n (\%) & $58(4.7)$ & $10(5.2)$ & 0.779 \\
Acute Respiratory Failure, n (\%) & $319(25.8)$ & $77(39.7)$ & $<0.001$ \\
Infections, n (\%) & $330(26.7)$ & $102(52.6)$ & $<0.001$
\end{tabular}

\section{D) Antithrombotic Therapies}

Anticoagulant Drugs, n (\%) $<0.001$

None

Any Anticoagulant

Type of Anticoagulant, n (\%)

LMWH

OAC

Antiplatelet Drugs, n (\%)

E) Other Treatments

ACEi/ARBs, n (\%)

Diuretics, n (\%)

Inotropic Drugs, n (\%)

Amiodarone, n (\%)

Propafenone/Flecainide, n (\%)

Sotalol, n (\%)
454 (36.7)

$782(63.3)$

0.361

312 (39.9)

$470(60.1)$

$515(41.7)$

$633(51.9)$

$925(75.8)$

$273(22.4)$

$381(31.2)$

$56(4.6)$

$16(1.3)$

F) Thromboembolic and Bleeding Risk

$\mathrm{CHA}_{2} \mathrm{DS}_{2}$-VASc, median [IQR]

$\mathrm{CHA}_{2} \mathrm{DS}_{2}$-VASc, mean (SD)

$\mathrm{CHA}_{2} \mathrm{DS}_{2}-\mathrm{VASc} \geq \mathbf{2}, \mathrm{n}(\%)$

$$
4[3-5]
$$

$4.28(1.68)$

1170 (94.7)
46 (23.7)

$148(76.3)$ 
Table 1: Baseline Characteristics at Admission in Sub-Intensive Unit (cont.)

\begin{tabular}{lccc}
\hline HAS-BLED, median $[\mathrm{IQR}]$ & $2[2-3]$ & $2[1-3]$ & $<0.001$ \\
HAS-BLED, mean (SD) & $2.38(1.08)$ & $2.07(0.95)$ & $<0.001$ \\
HAS-BLED $\geq \mathbf{3}, \mathrm{n}(\%)$ & $563(45.6)$ & $55(28.4)$ & $<0.001$
\end{tabular}

Legend: AF= Atrial Fibrillation; ACS= Acute Coronary Syndrome; AKI= Acute Kidney

Injury; $\mathrm{CKD}=$ Chronic Kidney Disease $\mathrm{COPD}=$ Chronic Obstructive Pulmonary Disease;

$\mathrm{eCVP}=$ Electrical Cardioversion Procedure; $\mathrm{pCVP}=$ Pharmacological Cardioversion

Procedure; IQR= Interquartile Range; LMWH= Low-Molecular Weight Heparin; OAC= Oral Anticoagulant; TIA= Transient Ischemic Attack. 
Table 2: Multivariate Logistic Regression Analysis for Composite Outcome Occurrence

\begin{tabular}{|c|c|c|c|}
\hline & OR & $95 \%$ CI & $\mathbf{p}$ \\
\hline Age (per year) & 1.03 & $1.01-1.05$ & 0.011 \\
\hline DBP (per mmHg) & 0.98 & $0.95-0.99$ & 0.023 \\
\hline Hypertension & 0.54 & $0.37-0.79$ & 0.001 \\
\hline Mitral Disease & 0.47 & $0.24-0.92$ & 0.028 \\
\hline ACS & 3.10 & $1.88-5.12$ & $<0.001$ \\
\hline Cardiogenic Shock & 10.06 & $5.37-18.84$ & $<0.001$ \\
\hline Septic Shock & 5.19 & $3.29-8.19$ & $<0.001$ \\
\hline Acute Respiratory Failure & 2.46 & $1.67-3.64$ & $<0.001$ \\
\hline \multicolumn{4}{|l|}{ Anticoagulant Drugs } \\
\hline None (reference) & - & - & - \\
\hline LMWH & 1.05 & $0.64-1.71$ & 0.857 \\
\hline $\mathrm{OAC}$ & 1.61 & $1.02-2.55$ & 0.040 \\
\hline Antiplatelet Drugs & 0.61 & $0.40-0.93$ & 0.021 \\
\hline
\end{tabular}

Legend: $\mathrm{ACS}=$ Acute Coronary Syndrome; $\mathrm{CI}=$ Confidence Interval; LMWH= LowMolecular Weight Heparin; OAC= Oral Anticoagulant; OR= Odds Ratio; TEE= Thromboembolic Events; TIA= Transient Ischemic Attack. 
Table 3: Guidelines Adherence, Clinical Risk Scores and Major Adverse Events

A) Multivariable-Adjusted Association between Guidelines Adherence and Major Adverse Events

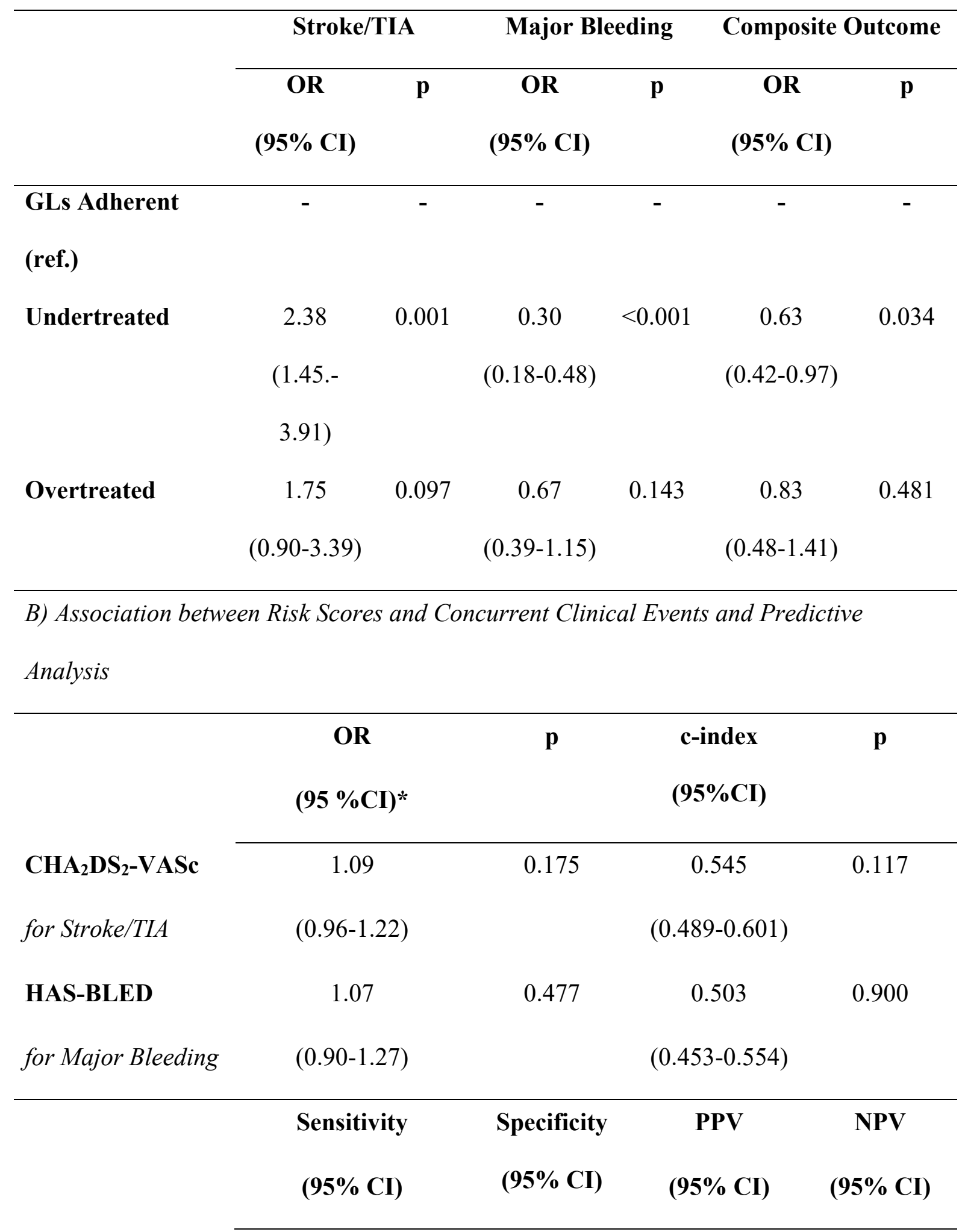




\begin{tabular}{|c|c|c|c|c|}
\hline $\mathrm{CHA}_{2} \mathrm{DS}_{2}-\mathrm{VASc}$ & $93.4 \%$ & $5.8 \%$ & $7.8 \%$ & $95.0 \%$ \\
\hline \multirow[t]{2}{*}{$\geq \mathbf{2}$ for Stroke/TIA } & $(90.9-99.0 \%)$ & $(4.6-7.1 \%)$ & $(6.4-9.2 \%)$ & (87.6- \\
\hline & & & & $98.1 \%)$ \\
\hline HAS-BLED $\geq 3$ & $46.6 \%$ & $57.1 \%$ & $10.0 \%$ & $91.3 \%$ \\
\hline for Major Bleeding & $(37.9-55.5 \%)$ & $(54.4-59.8 \%)$ & $(8.4-11.9 \%)$ & $(89.8-$ \\
\hline & & & & $92.5 \%)$ \\
\hline
\end{tabular}

Legend: *adjusted for type of AF and anticoagulant treatment; CI= Confidence interval; $\mathrm{GLs}=$ Guidelines; NPV= Negative predictive value; $\mathrm{OR}=$ Odds ratio; Positive predictive value; $\mathrm{TEE}=$ Thromboembolic Events; TIA $=$ Transient ischemic attack. 
Impact of atrial fibrillation in critically-ill patients admitted to a stepdown unit

Online-Only Supplementary Materials 
eFigure 1: Adherence to European Society of Cardiology Guidelines Recommendations

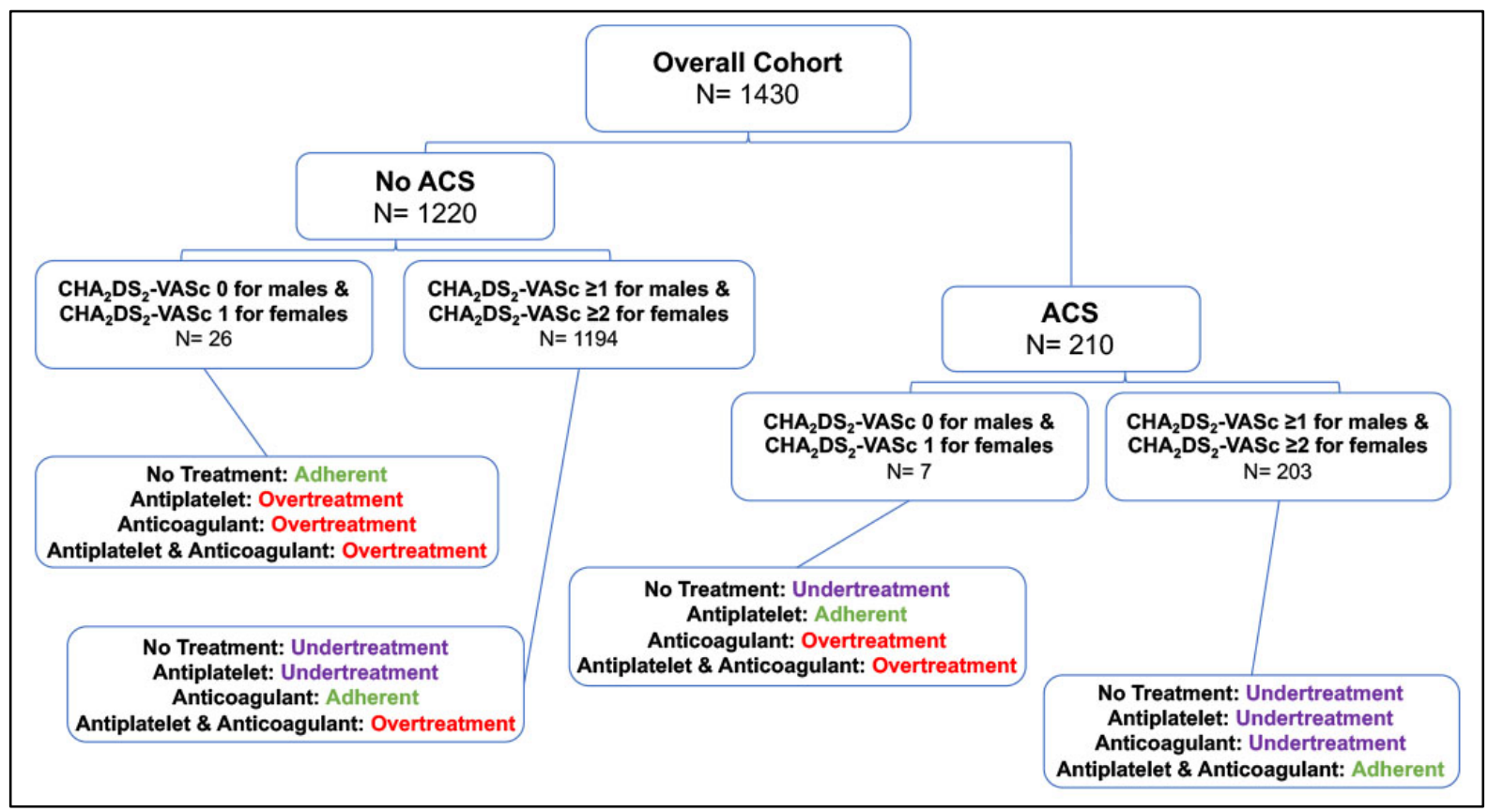

Legend: $\mathrm{ACS}=$ acute coronary syndrome. 
eFigure 2: Thromboembolic and Bleeding Risk according to Composite Outcome

\section{Occurrence}

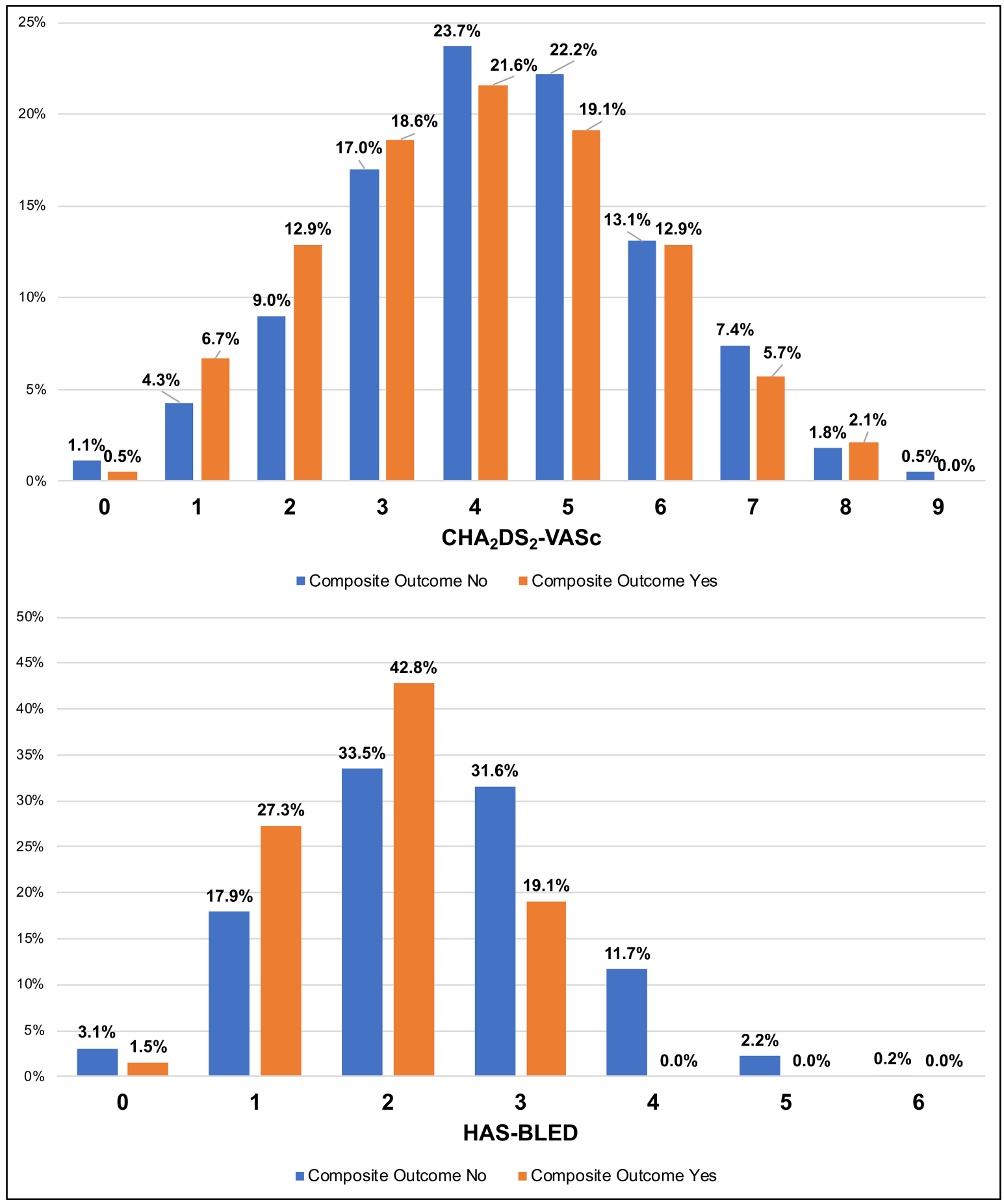


eTable 1: Multivariable-Adjusted Association between Guidelines Adherence and Major Adverse Events

\begin{tabular}{lllllll}
\hline & \multicolumn{2}{l}{ Stroke/TIA } & & Major Bleeding & & \multicolumn{2}{c}{ Composite Outcome } \\
\cline { 2 - 7 } & OR (95\% CI) & p & OR (95\% CI) & p & OR (95\% CI) & p \\
\hline GLs Adherent (ref.) & - & - & - & - & - & - \\
Undertreated & $2.38(1.45 .-3.91)$ & 0.001 & $0.30(0.18-0.48)$ & $<0.001$ & $0.63(0.42-0.97)$ & 0.034 \\
Overtreated & $1.75(0.90-3.39)$ & 0.097 & $0.67(0.39-1.15)$ & 0.143 & $0.83(0.48-1.41)$ & 0.481 \\
\hline
\end{tabular}

Legend: $\mathrm{CI}=$ Confidence interval; GLs= Guidelines; TIA= Transient ischemic attack. 
1 eTable 2: Major Adverse Events Rate according to Risk Scores

\begin{tabular}{lccc}
\hline CHA $_{2} \mathbf{D S}_{2}$-VASc & Stroke/TIA & HAS-BLED & Major Bleeding \\
\hline 0 & $\mathrm{n}(\%)$ & 0 & $\mathrm{n}(\%)$ \\
1 & $0(0.0)$ & 1 & $27(7.3)$ \\
2 & $4(6.1)$ & 2 & $41(8.2)$ \\
3 & $10(7.4)$ & 3 & $49(11.5)$ \\
4 & $15(6.1)$ & 4 & $10(6.2)$ \\
5 & $27(8.1)$ & $5(11.1)$ \\
6 & $24(7.7)$ & 5 & $0(0.0)$ \\
7 & $14(7.5)$ & 6 & \\
8 & $13(12.6)$ & & \\
9 & $3(11.5)$ & & \\
\hline
\end{tabular}

Legend: TIA $=$ Transient ischemic attack.

3

4 